\title{
BAL Tool in Flexible Manufacturing Systems *
}

\author{
Diego Perez, M.Carmen Ruiz, J.Jose Pardo, Diego Cazorla \\ Escuela Superior de Ingenieria Informatica \\ Univ. de Castilla-La Mancha \\ Campus Univ. s/n. 02071. Albacete, Spain. \\ \{Diego.Perez2, MCarmen.Ruiz, Juan.Pardo, Diego.Cazorla\}@uclm.es
}

\begin{abstract}
A tool for performance evaluation will be presented in this paper. The tool is named BAL and is based on the timed process algebra BTC incorporating resources as well. BAL begins by first making the syntactic analysis of the system specification, and then draws up its relevant transition graph by applying the rules of the operational semantics and solves a performance optimization problem relevant to optimize of the maximum throughout. Usage of BAL tool is illustrated on a Flexible Manufacturing Systems (FMS) to find a suitable number of resources/machines to maximize system throughput.
\end{abstract}

Keywords: Tool, Performance Evaluation, Available Resources, Timed Process Algebra, Flexible Manufacturing Systems

\section{Motivation}

Time-saving methods and increased performance are currently two of the overriding concerns in performance evaluation. Not so long ago, these features were considered important in critical systems, but now their relevance has become significant in all systems.

The aim of obtaining the best running time using the minimum amount of resources goes without question and it would be extremely beneficial to have a tool (in this case a tool based on formal methods) that is able to calculate the time needed to reach a certain state (or the final state for the total time), taking into account the time actions need for their execution, the time invested in process synchronizations and the fact that the (limited amount of) resources have to be shared by all the system processes, which could cause delays when resources are not available. Bearing in mind these factors, we have developed a timed process algebra, called BTC (for Bounded True Concurrency) [?], which allows us to evaluate the performance of a system as well as a tool called BAL (not an acronym) which is able to carry out this task automatically. BAL tool can be found in http://www.dsi.uclm.es/retics/bal.

BTC is based on CSP [?] and extends CSP syntax in order to consider the duration of actions by means of a timed prefix operator. Likewise, the operational

\footnotetext{
* This work has been partially supported by CICYT project TIN2009-14312-C02-02,
} and JCCM project PEII09-0232-7745 
semantics has also been extended to consider the context (resources) in which processes are executed. BTC is able to take into account the fact that system resources must be shared by all the processes. So, if there are more processes than resources then not all of them can be simultaneously executed. A process has to wait until it allocates the resources needed to continue its execution. This means that we can find two kinds of delays in the execution of a process: delays related to the synchronization of processes, and delays related to the allocation of resources. The former is usual in a (theoretical) concurrent context, but the latter is only taken into account if we consider a limited amount of available resources.

BAL tool is able to make the syntactic analysis of systems specified in BTC, build its relevant transition graph by applying the rules of the operational semantics and calculate the minimum time needed to reach the final state from the initial one.

This evaluation made by BAL can be used in two different ways. On the one hand, if we have a fixed number of resources we can ascertain the time needed to evolve from the initial state to the final one (or between states), so we can check different configurations for a system before using it, thus saving an immense amount of time and money. On the other hand, if we start with some specification, we can find the appropriate number of resources of any type that we need in order to fulfil some time requirements. We can even calculate the minimum number of resources needed to obtain the best performance from a system.

Apart from the usefulness of the analysis that BAL performs, the main advantage of this tool is the fact that it is capable of dealing with real-world systems of a considerable size. This has been achieved through painstaking work linking different implementation strategies.

In this paper, first BAL tool will be presented. Secondly, BAL is used in a case study which will highlight how BAL works and how the results obtained can be useful in Flexible Manufacturing Systems (FMS). Finally, conclusions and outlines for current and future work will be presented.

\section{BAL Tool}

With this formal resource-aware model the timed characteristics of the system are captured. The next step is concerned with carrying out performance evaluation: we want to be able to estimate the minimum time needed to reach a given state. By applying the rules of the operational semantics, we build a transition graph where we can abstract the information about actions and consider only the information about time (duration of actions). This graph is a weighted directed graph, where weights are always positive numbers, and the problem to solve is finding the shortest path from the initial node. Usually, the number of states in the transition graph for a real system is huge so the ability of a tool to perform this task automatically becomes relevant. With this idea in mind the BAL tool has been developed, which, moreover, has been improved with some 
other useful features: a syntactic analyzer and a graphical interface with a user assistant.

Thus, the BAL tool can be divided into three stages. First, a syntactic analyzer checks the specification of the system to be studied. If the system specification is correct, the second stage initiates. Here the tool builds the relevant transition graph by applying the rules of the operational semantics and lastly the minimum time needed to reach the final state from the initial one is calculated and the path obtained shown. Evidently, the most delicate part in the tool development has been that relative to graph analysis where it was necessary to join different algorithms of pruning, dynamic load balancing and parallelization. This is so, since one of the main aims is for the BAL tool to be able to deal with real systems and not be limited to toy examples. A general view of BAL tool can be found in Figure??.

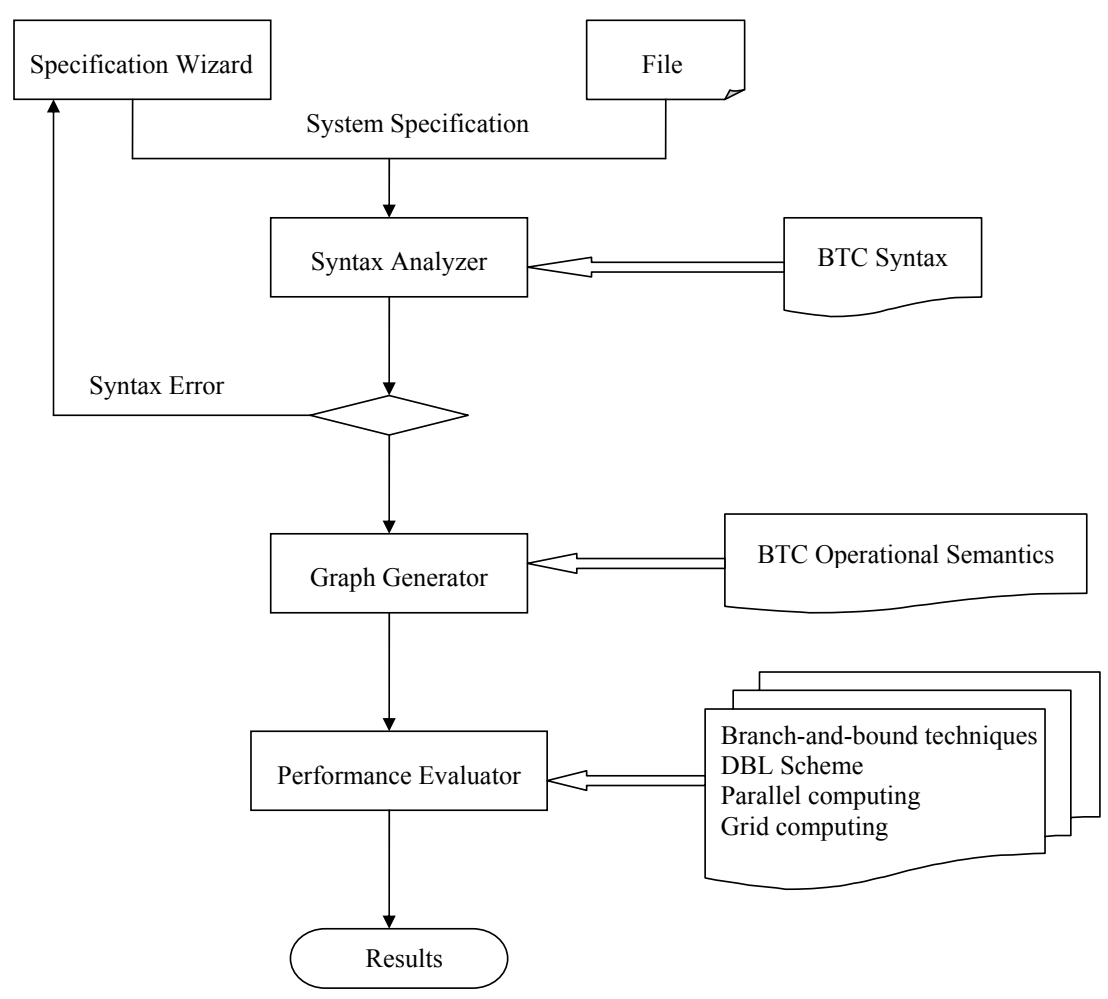

Fig. 1. Structure of the BAL tool 


\subsection{Graphical interface and assistant}

Besides being effective and efficient, the BAL tool should also be user-friendly and to that end, BAL includes a graphical interface and assistant. The BAL tool can thus be executed interactively in the command line or by making use of the graphical interface. Working in text mode it is necessary to supply a file with the specification of the system to be analyzed as an argument to the command $B A L$. The graphical interface requires little or no explanation because our standardization of the tools makes it easily recognisable for the user, and the new functions are also quite intuitive.

More attention needs to be given to the assistant, which has been developed to make specification tasks easier and avoid syntax errors whenever possible. This assistant consists of two main parts:

- Resource Assistant $\mathbf{Z}_{\mathbf{i}}$ : This helps to set the number of shared resources of any type in the system and the sets of actions that need these resources during their execution. Depending on the type of resource, the type of the action will be different. For preemptable resources the actions will be timed action while for non-preemptable ones the actions belonging to set $Z_{i}$ will be special actions to request and release the resource.

- Process Assistant: When the actions that make up the process must be supplied, it is easy to make a syntax error, so the assistant deals with the syntax details and the user just needs to give the action name and its type.

\section{Industrial Case Study - Flexible Manufacturing System}

A field where BTC has proved to be useful is in performance evaluation of Flexible Manufacturing Systems (FMS). A competitive manufacturing system is expected to be flexible enough to respond to small batches of customer demand. Due to the fact that the construction of any new production line requires a large investment, current production lines must be able to be reconfigured to keep up with increased frequency of new product designs. Therefore, in the development of a FMS, particular attention must be paid to the design phase, where the performance of the system has to be accurately evaluated so that the most appropriate number and type of resources are selected from the early stages of the design. In this context, the BAL tool is of great utility due to the fact that it allows us to check different configurations for a FMS before being set, or for future improvements.

Several FMS have been evaluated by means of BAL, in this section we will present a real system set in a local industry (Metalurgica y Plasticos de la Mancha, S.A [?]). In this system (Figure ??), pieces made up of two different parts (A and B) are created. These parts must be created by injecting the metal, filing it, vibrating or polishing it and then finally assembling it to form the final piece that will be packed for its distribution.

In this system we have seventeen types of resources, i.e., seven machines (M1-M7), two buffers (Buf1,Buf1), four conveyors $(C 1-C 2)$ and four robots 
$(R 1-R 4)$. The resources that model the machines are preemptable and the resources robot, buffer and conveyor are non-preemptable. For each we define a set consisting of the actions that need (at least) one resource of this type for their execution. For preemptable resources $(M 1-M 7)$ we define as follows:

$$
\begin{aligned}
& Z_{1}=\{\text { inject_ } A\} \quad Z_{3}=\{\text { file }\} \quad Z_{5}=\{\text { polish }\} \quad Z_{7}=\{\text { pack }\} \\
& Z_{2}=\{\text { inject_B }\} \quad Z_{4}=\{\text { vibrate }\} \quad Z_{6}=\{\text { assemble }\}
\end{aligned}
$$

which include the actions executed in any machine. The actions that need the non-preemptable resource

$$
\begin{array}{lll}
Z_{8}=\left\{r_{-} b 1\right\} & Z_{10}=\left\{r_{-} c 1\right\} & Z_{14}=\left\{r_{-} r 1\right\} \\
Z_{9}=\left\{r_{-} b 2\right\} & Z_{11}=\left\{r_{-} c 2\right\} & Z_{15}=\left\{r_{-} r 2\right\} \\
& Z_{12}=\left\{r_{-} c 3\right\} & Z_{16}=\left\{r_{-} r 3\right\} \\
& Z_{13}=\left\{r_{-} c 4\right\} & Z_{17}=\left\{r_{-} r 4\right\}
\end{array}
$$

The number of resources of any type is defined as:

$$
\mathcal{N}=\{2,4,2,2,2,4,2,8,9,12,12,1,1,1,2,2,1\}
$$

The BTC specification for this system is shown in Figure ??.

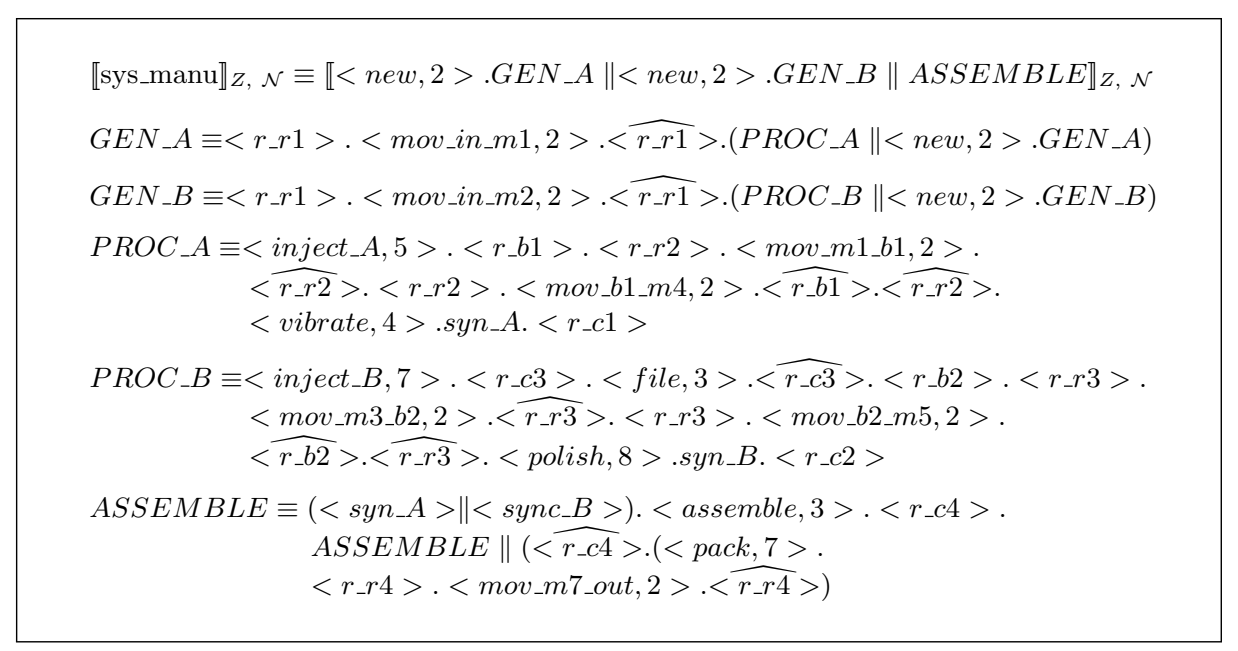

Fig. 2. Specification for the system

The performance evaluation of this system has been carried out using the BAL tool. Now, using the results obtained by the BAL tool, we can aim to work in either of two different ways: we can find the minimum number of resources needed to obtain a required system performance; or we can evaluate the performance of an established system to research any improvement. In this example, 


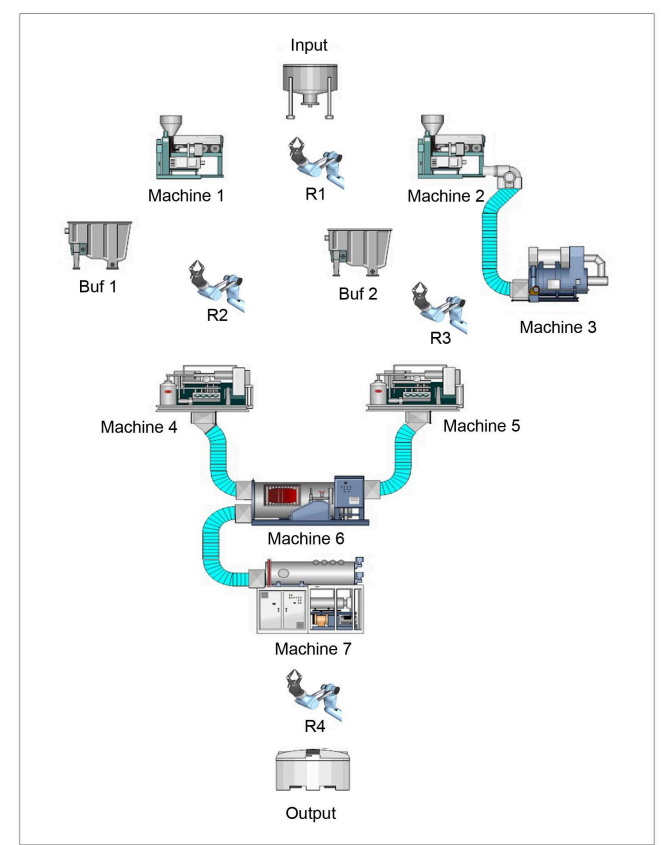

Fig. 3. Flexible Manufacturing System

we already have an established system, which we are going to study to see if it can be improved by achieving a better completion time i.e. a bigger number of completion parts per unit of time (throughput).

Different configurations (decreasing/increasing the number of robots, conveyor capacity and so on) have been studied and the conclusions are the following: After the first system evaluation a throughput of 0.137 is obtained, which means 13.70 pieces per 100 time units are obtained. Observing the behaviour of the system, we discovered that the R1 robot becomes a bottle neck, since there is a unique robot of this type and it must provide raw pieces for 6 machines $(2$ $\mathrm{M} 1+4 \mathrm{M} 2$ ). The system is analyzed again but now with $2 \mathrm{R} 1$ robots and surprisingly no improvement is observed. In fact a throughput of 0.119 is obtained which is inferior to the previous one. This problem improves partially when increasing up to 4 the number of robots R1, but thrown data (throughput 0.14) are not significant enough to be worth the trouble of increasing the number of robots to this number. The problem arises because the storage capacity of the C3 conveyor is not prepared for this modification. By changing this capacity $(\mathcal{N}=\{2,4,2,2,2,4,2,8,9,12,12,10,1,2,2,2,1\})$ an important improvement is achieved, 23 pieces per 100 time units being obtained (throughput 0.23 ). Thus, we propose using $2 \mathrm{R} 1$ robots and increasing the $\mathrm{C} 3$ conveyor capacity up to 50 elements. Another important conclusion obtained is that with an extra M7 machine the system produces twenty percent more parts per time unit. 


\section{Conclusions and Future Work}

In this work we have presented the BAL tool which has been developed to carry out performance evaluation. Besides this main goal, BAL tool has been improved using an syntactic analyzer and a graphical interface including a help assistant when the system specification is written. Therefore, the BAL tool consists of the following parts:

- Graphical interface

- Assistant for system specification

- System analyzer

- Graph generator

- Performance Evaluator

A case study has been made to show how the results obtained by BAL can be used; with a fixed number of resources we can ascertain the time needed to evolve from the initial state to the final one, so we can check different configurations for a system before using it, hence saving a huge amount of time and money. We can even calculate the minimum number of resources needed to obtain the best performance from a system.

Currently, our work is focused on two different lines. The first one is relative to improving the tool and the second one focuses on its application.

As stated above, the tool uses parallel and grid computers with distributed memory, but with the recent improvements in hardware architecture where multicore machines are a reality, we have decided to take advantage of such architectures and used threads which will communicate by means of shared memory.

The main field where BTC is proving its worth is in the performance evaluation of FMS. A competitive manufacturing system is expected to be flexible enough to respond to small batches of customer demand. Due to the fact that the construction of any new production line is a large investment, current production lines must be able to be reconfigured to keep up with increased frequency of new product designs. Therefore, in the development of a FMS, particular attention must be paid to the design phase, where the performance of the system has to be accurately evaluated so that the most appropriate number and type of resources are selected from the initial design stages.

In this context, BTC and BAL are of great utility given that they allow us to check different configurations for a flexible manufacturing system before being established or for future improvements. 